\title{
Moxibustion improves ovarian function based on the regulation of the androgen balance
}

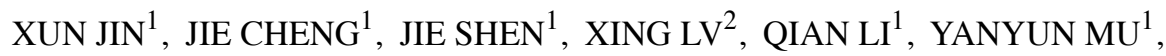 \\ HUA BAI $^{1}$, YAN LIU ${ }^{1}$ and YOUBING XIA ${ }^{3}$ \\ ${ }^{1}$ College of Acupuncture and Massage, Nanjing University of Chinese Medicine, Nanjing, Jiangsu 210023; \\ ${ }^{2}$ Central Research Institute of Shanghai Pharmaceuticals Holding Co., Ltd., Shanghai 200020; \\ ${ }^{3}$ Traditional Chinese Medicine Department, The Affiliated Hospital of Xuzhou Medical \\ University, Xuzhou Medical University, Xuzhou, Jiangsu 221006, P.R. China
}

Received August 1, 2020; Accepted June 18, 2021

DOI: 10.3892/etm.2021.10664

\begin{abstract}
The effect of androgens on follicular development and female reproduction has become an active research topic. Moxibustion is a Traditional Chinese Medicine therapy that has been reported to be able to prevent and treat numerous ovary-related problems. However, studies on the effect of moxibustion for diminished ovarian reserve (DOR) on androgen balance are still lacking. The present study aimed to assess the efficacy of moxibustion intervention prior to disease onset and at the early stage of disease in a rat model of DOR and explore the mechanisms of its effect on ovarian function. A total of 32 rats were randomly divided into four groups: Blank group, Model group (a drug-induced model of DOR), Moxibustion group 1 and Moxibustion group 2 . Moxibustion was performed on the BL23 and RN4 acupoints of female rats daily for a total of 20 days (once a day, five times a week for a total of 4 weeks). The two moxibustion groups were established with different intervention times: One group was subjected to pre-disease intervention and the other group to early-disease intervention. The ovarian function was evaluated by detecting anti-Mullerian hormone $(\mathrm{AMH})$, follicle-stimulating hormone (FSH), estradiol (E2), testosterone (T), dehydroepiandrosterone (DHEA), dihydrotestosterone (DHT) and androgen receptor (AR) levels in the serum or the ovary samples. To further investigate the downstream regulatory factors for AR after moxibustion treatment for pre-disease or early-disease intervention, FSH receptor (FSHR) and microRNA (miR)-125b expression in ovaries were also analyzed. The results indicated that
\end{abstract}

Correspondence to: Professor Youbing Xia, Traditional Chinese Medicine Department, The Affiliated Hospital of Xuzhou Medical University, Xuzhou Medical University, 99 Huaihai West Road, Xuzhou, Jiangsu 221006, P.R. China

E-mail: xybd1968@sina.com

Key words: moxibustion, diminished ovarian reserve, androgen balance, pre-disease intervention, early disease intervention
AMH and DHT levels were reduced in the model group compared with those in the blank group, while FSH, T and DHEA levels were increased. AMH and DHT levels were increased in Moxibustion group 1 compared with those in the model group, while FSH, T and DHEA levels were reduced. There was no difference in E2 levels between Moxibustion group 1 and the model group. Compared with that in the model group, the AR content in the ovary was increased in Moxibustion group 1. There was no difference in FSHR mRNA in the ovaries between Moxibustion group 1 and the model group. miR-125b levels were significantly increased in Moxibustion group 1 as compared with those in the model group. Furthermore, AMH and DHT levels were increased in Moxibustion group 2 compared with those in the model group, while FSH, T and DHEA levels were reduced. E2 levels were significantly decreased in Moxibustion group 2 compared with those in the model group. The relative mRNA expression of AR, FSHR and miR-125b was decreased following establishment of the model. Compared with that in the model group, the AR content in the ovary was increased in Moxibustion group 2. In comparison with the blank and model groups, the FSHR content in the ovary of Moxibustion group 2 was significantly increased. miR-125b levels were not obviously altered in Moxibustion group 2 as compared with those in the model group. In addition, there was no significant difference in AMH, FSH, T and DHEA levels between the two moxibustion groups. E2 and DHT levels were higher in Moxibustion group 1 than in Moxibustion group 2. There was no difference in AR mRNA expression between the two moxibustion groups. FSHR mRNA levels were lower in Moxibustion group 1 than in Moxibustion group 2, while miR-125b mRNA levels were higher in Moxibustion group 1 than in Moxibustion group 2. In conclusion, the present study suggested that moxibustion intervention prior to disease onset and at the early disease stage was able to improve ovarian function via modulation of the AR-mediated stable equilibrium of androgens. However, the effects and mechanisms of moxibustion intervention for pre-disease and early-disease intervention of DOR appear to be different. The appropriate duration of treatment and the time-effect relationship require to be further studied. 


\section{Introduction}

Diminished ovarian reserve (DOR) refers to abnormal ovarian function caused by various factors prior to the age of 40 years and results in associated symptoms, which is the occult abnormal ovarian function period prior to ovarian failure amenorrhea $(1,2)$. Without any timely and effective intervention, the ovaries of patients with DOR may gradually shrink within 1 to 6 years, leading to premature ovarian failure (POF) (3).

At present, the effect of androgens on follicular development and female reproduction is under active investigation. Androgens, including dehydroepiandrosterone (DHEA), DHEA-sulfate (DHEA-S), androstenedione (A4), testosterone (T) and dihydrotestosterone (DHT), are important hormones in the female endocrine and reproductive systems. DHEA, DHEA-S and A4 must be bioconverted to T or DHT to have a physiological role $(4,5)$. It has been suggested that the androgen effect is generated when androgens are converted to estradiols under the action of aromatase in granulosa cells; the produced estradiols then act on estradiols receptors. Further studies indicated that androgen itself positively affects follicular development and T or DHT will eventually bind to the androgen receptor (AR) to activate downstream signaling pathways, such as follicle-stimulating hormone receptor (FSHR) and microRNA (miRNA/miR)-125b to induce the corresponding biological effect $(6,7)$.

Moxibustion is a Traditional Chinese Medicine (TCM) therapy. It uses Folium Artemisiae argyi to produce moxibustion materials, generating heat upon combustion to stimulate acupoints or specific body surface sites, thereby achieving disease prevention and treatment. Increasing evidence has demonstrated that moxibustion is able to improve ovarian function (8-12). It has been reported that the early use of moxibustion is able to generate a favorable stress response to resist or reduce the subsequent disease and delay the degradation of normal tissues (13). To date, studies on the mechanisms underlying the improvement in ovarian function by moxibustion have mainly focused on the hypothalamic-pituitary-ovarian axis (HPOA) $(14,15)$. There is a lack of studies focusing on androgen balance and AR-mediated signaling pathways. It remains elusive whether the protective roles and mechanisms underlying moxibustion intervention on ovarian function prior to the onset or at early stages of DOR are different.

In the present study, moxibustion was performed on the BL23 and RN4 acupoints of female rats daily for a total of 20 times. For this, two moxibustion groups were established with different intervention times: One group was subjected to pre-disease intervention and the other group to early-disease intervention. The ovarian function was evaluated based on the levels of several hormones related to ovarian function, particularly androgens. To further investigate the downstream regulatory factors of AR after moxibustion intervention prior to the onset or at early stages of POF, FSHR and miR-125b expression in ovaries were also analyzed. The present study focused on rat physiology, providing a strong foundation and a complementary understanding of moxibustion application as both physiological and pathological research.

\section{Materials and methods}

Animal experiment. A total of 32 female Sprague-Dawley rats weighing 220-280 $\mathrm{g}$ and aged 8-12 weeks were provided by Shanghai Super-B\&K Laboratory (Animal production license no. SYXK, Shanghai 2017-0002). The rats were maintained under a normal 12-h light/dark cycle at $22 \pm 2^{\circ} \mathrm{C}$ with $50-70 \%$ relative humidity. The rats were allowed to adapt to the surrounding environment for two weeks. Animals with a regular estrus cycle and the same estrus stage were detected through vaginal smears. Rats were housed with four animals per cage and food pellets and water provided ad libitum.

A total of 32 rats were randomly divided into four groups (Fig. 1): Blank group (normal saline was administered), Model group (rat model was established via intragastric administration of tripterygium glycosides), Moxibustion group 1 (rat model was established after 4 weeks of moxibustion treatment, making this the pre-disease intervention group) and Moxibustion group 2 (rats were treated with tripterygium glycosides and moxibustion for 2 weeks, followed by another 2 weeks of moxibustion treatment, making this the early-disease intervention group). The day of grouping was recorded as day 1. All experimental procedures were approved by the Animal Care and Use Committee of Nanjing University of Chinese Medicine (Nanjing, China; no. ACU170709).

Observation of estrus stages. A thin cotton swab dipped in saline was gently inserted into the vagina of the rats and used to scrape around the cervix of the rats. The vaginal secretions were evenly smeared onto slides. After $15 \mathrm{~min}$ of fixation, the vaginal secretions were subjected to Pap staining and cytological examination was performed under the microscope. The observation of estrus stages began on the 43rd day of the experiment and was continually performed once a day for 14 consecutive days and ended on the 56th day. A summary of the regularity of the estrus stages in the rats was generated at the end of the study. The estrus stages of normal female rats lasted for 4-5 days, including the proestrus, estrus, metestrus and diestrus stages (16). 'Extension of estrus stages' is defined as estrus stages that lasted $>5$ days and 'disorder of estrous stages' was defined as estrous cycles that could not be observed or continued to be diestrus.

Model construction methods. The DOR model was established by referring to the methods reported in the literature $(17,18)$ and began on the 29th day of the experiment (Fig. 1). Normal saline $(2 \mathrm{ml})$ was administered once a day by gavage in the blank group, while $2 \mathrm{ml}$ of tripterygium glycosides (Shanghai Fudan Fuhua Pharmaceutical Co., Ltd.; no. Z31020415) was administered at $75 \mathrm{mg} / \mathrm{kg} /$ day once a day IG for 14 consecutive days in the other groups. The whole modeling process was uneventful and no death occurred. If the vaginal smear of the rats exhibited no change of the sexual cycle in $>2$ sexual cycles or prolonged estrus stage of $>6$ days, the model was considered to be successfully established.

Moxibustion application. The acupoint location was determined referring to 'Experimental Acupuncture' (19). Moxibustion was applied to the bilateral 'Shenshu' acupoint 


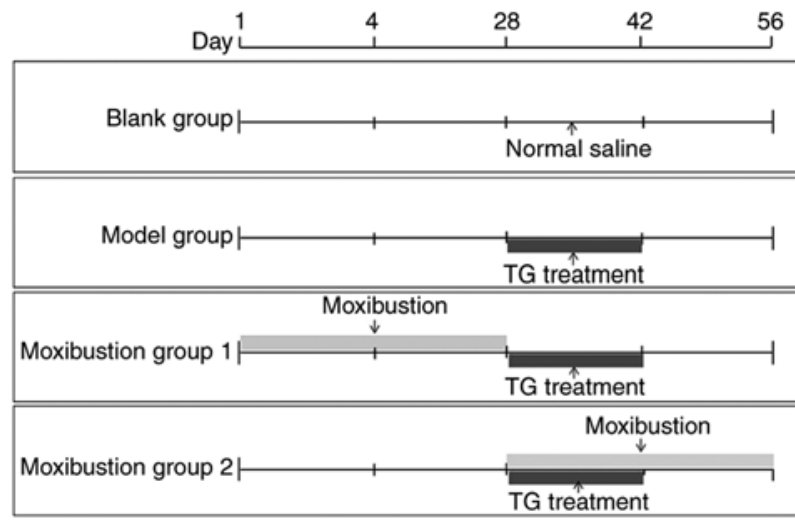

Figure 1. Flow diagram of the study. TG, tripterygium glycosides.

(BL 23, $7 \mathrm{~mm}$ lateral to the spinous process of the second lumbar spine) in the prone position and the 'Guanyuan' acupoint (RN 4, $25 \mathrm{~mm}$ below the navel) in the supine position daily. The specific moxibustion methods were performed as follows: Two assistants performed the procedure. One researcher was responsible for holding the animals with its hand, and the other researcher performed the moxibustion. The rat was blindfolded. The neck of the rats were first touched to quieten them and then the rats gently held in the hand without anesthetization $(20,21)$. Hair on the treatment area was shaved and Vaseline was applied. A grain-sized moxa cone (5 mg of pure moxa cone with a base of 3.0-3.5 mm and a height of 4-5 $\mathrm{mm}$ ) was placed on the acupoints using tweezers and ignited with a match (the burning time of each cone was $8-10 \mathrm{sec}$, with a temperature of $48-52^{\circ} \mathrm{C}$ at the acupoints). A new moxa cone was applied when the prior one was completely burned. Seven grain-sized moxa cones were applied at each acupoint per treatment for a total of 20 days (once a day, five times a week for a total of 4 weeks). The duration of one treatment session for each rat was $\sim 3 \mathrm{~min}$. The moxibustion in moxibustion group 1 and moxibustion group 2 began on the 1st day and the 29th day of the experiment, respectively (Fig. 1). The rats in the non-moxibustion group were also fixed with the assistant's hand and blindfolded synchronously. During and after the procedure, the wellbeing of the rats was assessed by observing their behavior and attempting to detect any moxibustion ulcer formation. It was observed that the rats were quiet when treated with moxibustion: No foot lifting and licking, no back arching, no tremor or spasm and regular breathing. Following moxibustion, there were no moxibustion ulcers in any of the rats with regular food and water consumption (22).

Hormone assays. Hormones were detected at the end of the experiment to ensure that the blood samples were collected at the same stage of the menstrual cycle. Whole blood samples $(2 \mathrm{ml})$ were harvested by a retro-orbital puncture after animals were anesthetized with a single intraperitoneal injection of $7 \%$ chloral hydrate $(350 \mathrm{mg} / \mathrm{kg})$ from eight rats in each group. The animals were sacrificed 10 min after injection of chloral hydrate by cervical dislocation. Then, the animals were sacrificed by cervical dislocation. The samples were centrifuged at $1,500 \mathrm{xg}$ for $20 \mathrm{~min}$ at room temperature and the supernatants were collected. Serum anti-mullerian hormone (AMH), FSH, estradiol (E2), T, DHEA and DHT were detected using the rat AMH ELISA kit (cat. no. m1060605), FSH ELISA kit (cat. no. m1002872), E2 ELISA kit (cat. no. ml002871), T ELISA kit (cat. no. ml003368), DHEA ELISA kit (cat. no. ml003097) and DHT ELISA kit (cat. no. ml002998), respectively (Shanghai Mlbio Co., Ltd.). The optical density of the ELISA plates was measured at $450 \mathrm{~nm}$ by a microplate reader.

Reverse transcription-quantitative (q)PCR. The fresh ovarian tissue was separated from six sacrificed rats in each group and they were used for RNA analysis. Total RNA was extracted using TRIzol ${ }^{\circledast}$ reagent (Invitrogen; Thermo Fisher Scientific, Inc.) and was reverse transcribed using a First-strand cDNA Synthesis kit (Invitrogen; Thermo Fisher Scientific, Inc.). miRNA was harvested using the miRcute miRNA isolation kit (Tiangen Biotech Co., Ltd.) and was reverse transcribed using stem-loop primers and the Thermo First-strand cDNA Synthesis kit (Thermo Fisher Scientific, Inc.). The following primers were used: AR forward, 5'-GAG ACGACACGATGGACAATT-3' and reverse, 5'-GCGGAA GGGAAACAGAAGTAT-3'; FSHR forward, 5'-TGAATG ATTAAGAGGGACAAGC-3' and reverse, 5'-AAGCCCAGA TTTACAGGACAG-3'; Rat 18S rRNA forward, 5'-GAATTC CCAGTAAGTGCGGGTCATA-3' and reverse, 5'-CGAGGG CCTCACTAAACCATC-3'. miR-125b forward, 5'-CGGGCT CCCTGAGACCCTAA-3' and reverse, 5'-CAGCCACAA AAGAGCACAAT-3', miRNA/U6 forward, 5'-CCTGCT TCGGCAGCACA-3' and reverse, 5'-AACGCTTCACGA ATTTGCGT-3'. The qPCR Fluorescence Quantitation kit (Applied Biosystems; Thermo Fisher Scientific, Inc.) and the CFX384 Touch $^{\mathrm{TM}}$ Real-Time PCR Detection System (Bio-Rad Laboratories, Inc.) were used for detecting AR and FSHR expression. The TaqMan ${ }^{\circledR}$ Fast Advanced Master Mix (Thermo Fisher Scientific, Inc.) and the CFX384 Touch ${ }^{\mathrm{TM}}$ Real-Time PCR Detection System (Bio-Rad Laboratories, Inc.) were used for detecting miRNA-125b expression. The reaction for detecting AR and FSHR expression was performed in a 10- $\mu 1$ system $(5,0.2,0.2,1,0.2$ and $3.4 \mu 1$ of 2X ChamQ SYBR qPCR Master Mix (Applied Biosystems; Thermo Fisher Scientific, Inc.), forward primer $10 \mu \mathrm{M}$, reverse primer $10 \mu \mathrm{M}$, template DNA, 50X ROX Reference Dye 1, nuclease-free $\mathrm{H}_{2} \mathrm{O}$, respectively). The program was set to two steps for real-time quantitation: Initial denaturation was performed at $95^{\circ} \mathrm{C}$ for $10 \mathrm{~min}$. Subsequently, each denaturation was $95^{\circ} \mathrm{C}$ for $15 \mathrm{sec}$, followed by an annealing elongation at $63^{\circ} \mathrm{C}$ for $30 \mathrm{sec}$. The above steps comprised one cycle and there were 40 cycles in total. The reaction for detecting miR-125b expression was performed in a $20-\mu 1$ system [SDW $7.5 \mu$, TaqMan ${ }^{\circledR}$ Fast Advanced Master Mix (2X) $10.0 \mu \mathrm{l}$, miRNA-125b forward $0.5 \mu \mathrm{l}$, universal miRNA-125b reverse $0.5 \mu \mathrm{l}$, universal TaqMan probe $0.5 \mu \mathrm{l}$ and template DNA $1.0 \mu \mathrm{l}]$. The program was set to two steps for real-time quantitation: Initial denaturation was performed at $50^{\circ} \mathrm{C}$ for $2 \mathrm{~min}$. Subsequently, each denaturation was $95^{\circ} \mathrm{C}$ for $5 \mathrm{sec}$, followed by an annealing elongation at $60^{\circ} \mathrm{C}$ for $25 \mathrm{sec}$. The above steps comprised one cycle and there were 40 cycles in total. The fluorescence value was read during each extension stage and the dissolution curve was prepared after the end of the cycle. Each sample was analyzed in 

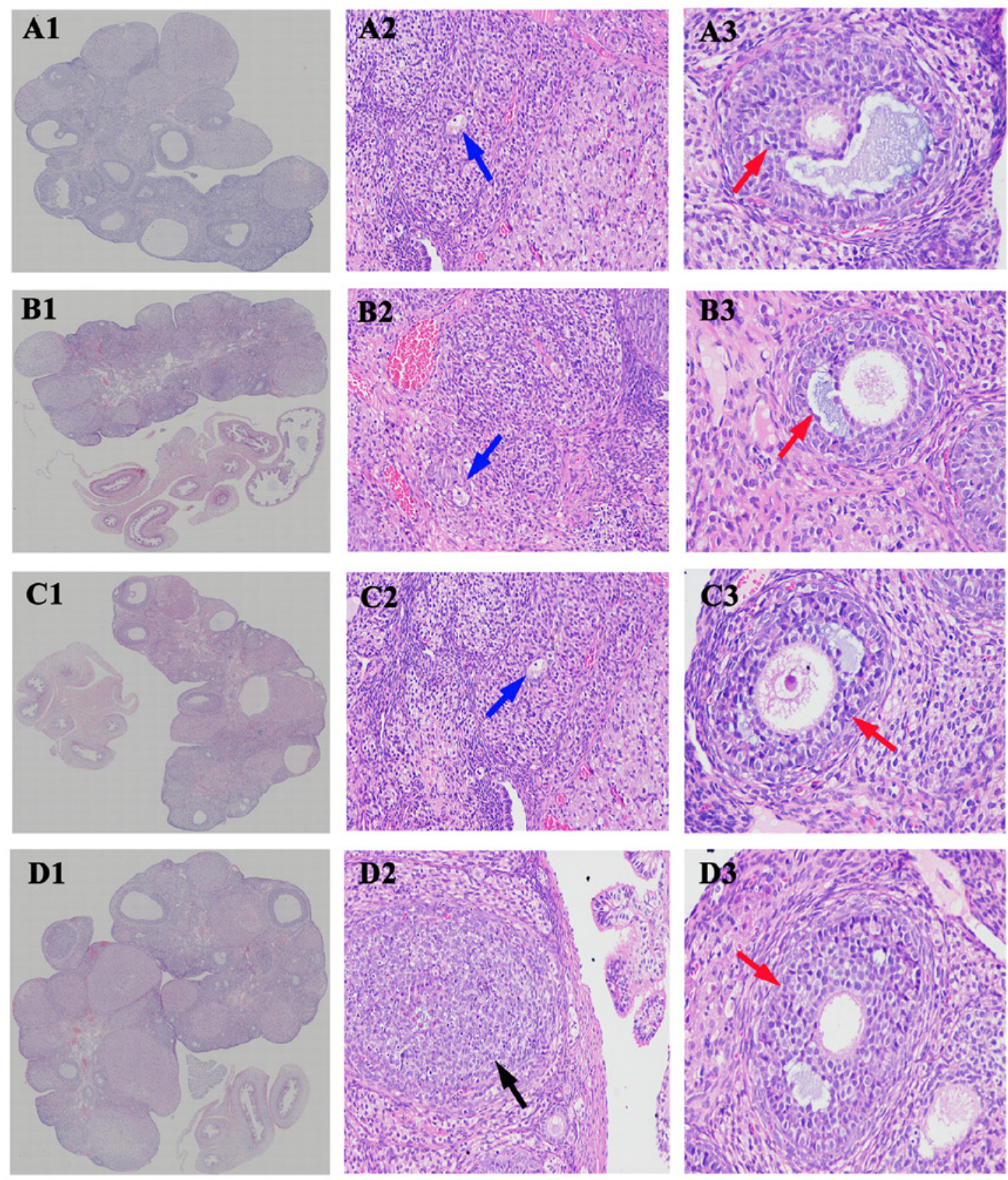

Figure 2. Moxibustion stimulation improves tripterygium glycoside-induced histopathological changes in rats. Histopathology images of ovaries from (A) the blank group, (B) model group, (C) moxibustion group 1 and (D) moxibustion group 2 (H\&E staining; original magnification, x20, x200 and x400, in column 1 , 2 and 3 , respectively). The blue, red and black arrows indicate atretic follicles, mature follicular cells and the ovarian granuloma cells, respectively.

triplicate and ultimately, the relative expression levels of each gene were calculated with the $2^{-\Delta \Delta C q}$ method (23).

Histopathology. The right ovaries from eight sacrificed rats in each group were fixed in $4 \%$ paraformaldehyde for histopathological examination. After fixation, each tissue sample was routinely processed and embedded in paraffin. Subsequently, they were sectioned at $4 \mu \mathrm{m}$ thickness and stained with $\mathrm{H} \& \mathrm{E}$ for observation. Ovarian follicles were classified into the primordial follicle (an oocyte surrounded by one layer of flattened graunlosa cells), the primary follicle (an oocyte surrounded by one layer of cuboidal graunlosa cells), the secondary follicle (two or three layers of cuboidal granulose cells with no antral space) and antral follicle (more than four layers of granulosa cells with one or more independent antral spaces) (16). Atretic follicles contained $\geq 20$ apoptotic granulosa cells, disorganized granulosa cells, fragmentation of the oocyte nucleus or a degenerating oocyte. The number of primary follicles, secondary follicles, antral follicles and atretic follicles was calculated according to the morphological characteristics of follicles in the different groups.

Statistical analysis. Values are expressed as the mean \pm standard deviation. The statistical analysis was performed by SPSS 22.0 statistical software (IBM Corporation). Statistical significance was determined by one-way ANOVA with Tukey's post-hoc test. The difference in estrus stages among the different groups was analyzed by 
Table I. Number of various types of follicle per ovary in the different groups.

\begin{tabular}{lcccc}
\hline Group & Primary follicles & Secondary follicles & Antral follicles & Atretic follicles \\
\hline Blank & $7.00 \pm 3.09$ & $5.38 \pm 2.06$ & $3.37 \pm 0.89$ & $5.00 \pm 1.15$ \\
Model & $4.50 \pm 2.97$ & $3.50 \pm 1.26^{\mathrm{a}}$ & $2.71 \pm 1.44$ & $9.12 \pm 2.45^{\mathrm{b}}$ \\
Moxibustion 1 & $4.90 \pm 2.02$ & $5.57 \pm 1.91^{\mathrm{c}}$ & $3.50 \pm 1.96$ & $7.00 \pm 3.15^{\mathrm{c}}$ \\
Moxibustion 2 & $7.33 \pm 3.25^{\mathrm{c}, \mathrm{d}}$ & $4.67 \pm 1.94$ & $4.89 \pm 5.14$ & $5.44 \pm 1.69^{\mathrm{c}}$ \\
$\chi^{2}$ & 4.370 & 4.099 & 1.577 & 10.813 \\
P-value & 0.007 & 0.010 & 0.204 & 0.000 \\
\hline
\end{tabular}

Values are expressed as the mean \pm standard deviation. ${ }^{a} \mathrm{P}<0.05$, ${ }^{b} \mathrm{P}<0.001$ vs. blank group; ${ }^{\mathrm{c}} \mathrm{P}<0.05$ vs. model group; ${ }^{\mathrm{d}} \mathrm{P}<0.05$ vs. moxibustion group 1 .

Table II. Changes in estrus stages in the rats.

\begin{tabular}{lcccc}
\hline Group & Total number & Normal & Extension & Disorder \\
\hline Blank & 8 & 7 & 1 & 0 \\
Model $^{\text {a }}$ & 8 & 1 & 4 & 3 \\
Moxibustion 1 $_{\text {Moxibustion 2 }}$ & 8 & 3 & 4 & 1 \\
\end{tabular}

${ }^{\mathrm{a}} \mathrm{P}<0.05$ vs. blank group. Extension is defined as estrus stages that lasted $>5$ days. Disorder is defined as estrus cycles that could not be observed or continued to be diestrus.

using the $\chi^{2}$ test. $\mathrm{P}<0.05$ was considered to indicate statistical significance.

\section{Results}

Moxibustion stimulation improves tripterygium glycoside-induced histopathological changes in rats. As presented in Fig. 2 and Table I, compared with the blank group, the number of atretic follicles was significant increased in the model group $(5.00 \pm 1.15$ vs. $9.12 \pm 2.45$, $\mathrm{P}<0.001$ ), while the number of mature follicular cells was decreased and the ovarian granuloma cells were observed to be undergoing significant apoptosis, with rare corpus luteum. Compared with the model group, the number of atretic follicles in both moxibustion group 1 and moxibustion group 2 were significantly reduced $(9.12 \pm 2.45$ vs. $7.00 \pm 3.15, \mathrm{P}<0.05$ and $9.12 \pm 2.45$ vs. $5.44 \pm 1.69, \mathrm{P}<0.05$, respectively). The number of mature follicular cells was increased with enhanced corpus luteum and decreased apoptotic ovarian granuloma cells as compared with the model group.

Changes in the estrus stages in rats. As presented in Table II, the estrus stage of rats in the blank group was regular, while the stages were expanded to different degrees in the model group, moxibustion group 1 and moxibustion group 2 in comparison to the blank group, particularly in the model group $(\mathrm{P}<0.05)$. A portion of the rats in the model group continued to exhibit diestrus. No significant difference was observed between the moxibustion group and the blank group. No significant difference was observed between the moxibustion group and the model group.

Moxibustion attenuates DOR-associated changes in AMH, FSH and E2 in serum. The serum concentrations of AMH, FSH and E2 were analyzed to evaluate ovarian function. As presented in Fig. 3A, AMH levels were significantly reduced after modeling compared with the blank group $(2.24 \pm 0.18$ vs. $2.94 \pm 0.30 \mathrm{pmol} / 1, \mathrm{P}<0.01)$, while they were increased in Moxibustion group 1 and Moxibustion group 2 compared with the model group $(2.59 \pm 0.35 \mathrm{vs} .2 .24 \pm 0.18 \mathrm{pmol} / 1, \mathrm{P}=0.153$ and $2.74 \pm 0.30$ vs. $2.24 \pm 0.18 \mathrm{pmol} / 1, \mathrm{P}<0.05$, respectively). There was no difference in $\mathrm{AMH}$ levels between the 2 moxibustion groups.

As presented in Fig. 3B, FSH levels significantly increased after establishing the model (1,725.32 \pm 138.96 vs. $3,616.87 \pm 262.46 \mathrm{pg} / \mathrm{ml}, \mathrm{P}<0.001)$. FSH levels were reduced in Moxibustion group 1 and Moxibustion group 2 compared with the model group $(3,142.52 \pm 290.95$ vs. $3,616.87 \pm 262.46 \mathrm{pg} / \mathrm{ml}$, $\mathrm{P}<0.05$ and $3,109.37 \pm 317.38$ vs. $3,616.87 \pm 262.46 \mathrm{pg} / \mathrm{ml}, \mathrm{P}<0.01$, respectively). There was no difference in FSH levels between the 2 moxibustion groups. As indicated in Fig. 3C, serum E2 levels were significantly increased in the model group compared with the blank group (41.45 \pm 3.88 vs. $22.14 \pm 3.24 \mathrm{pmol} / \mathrm{l}, \mathrm{P}<0.001)$; they decreased significantly in Moxibustion group 2 compared with the model group $(30.53 \pm 3.64$ vs. $41.45 \pm 3.88 \mathrm{pmol} / 1$, $\mathrm{P}<0.01)$. Significant differences in E2 levels were observed between Moxibustion group 1 and Moxibustion group 2 (40.30 \pm 6.16 vs. $30.53 \pm 3.64 \mathrm{pmol} / \mathrm{l}, \mathrm{P}<0.01)$.

Moxibustion stimulation attenuates DOR-associated changes in T, DHEA and DHT levels in serum. As T, DHEA and DHT are the three major androgens and changes in their levels affect ovarian function, their serum levels were analyzed to investigate whether there is an association between androgen balance and ovarian function after moxibustion treatment. As illustrated in Fig. 4A and B, T and DHEA levels significantly increased after establishing the model (101.24 \pm 9.76 vs. $230.80 \pm 21.03 \mathrm{pg} / \mathrm{l}, \mathrm{P}<0.001$ and $19.98 \pm 1.63$ vs. $28.49 \pm 2.63 \mathrm{ng} / 1, \mathrm{P}<0.001$, respectively). $\mathrm{T}$ levels were lower in Moxibustion group 1 and Moxibustion group 2 compared with the model group $(209.10 \pm 16.22$ vs. $230.80 \pm 21.03 \mathrm{pg} / 1$, $\mathrm{P}=0.078$ and $207.75 \pm 16.77$ vs. $230.80 \pm 21.03 \mathrm{pg} / \mathrm{l}, \mathrm{P}=0.064$, respectively). No significant difference in $\mathrm{T}$ levels was 

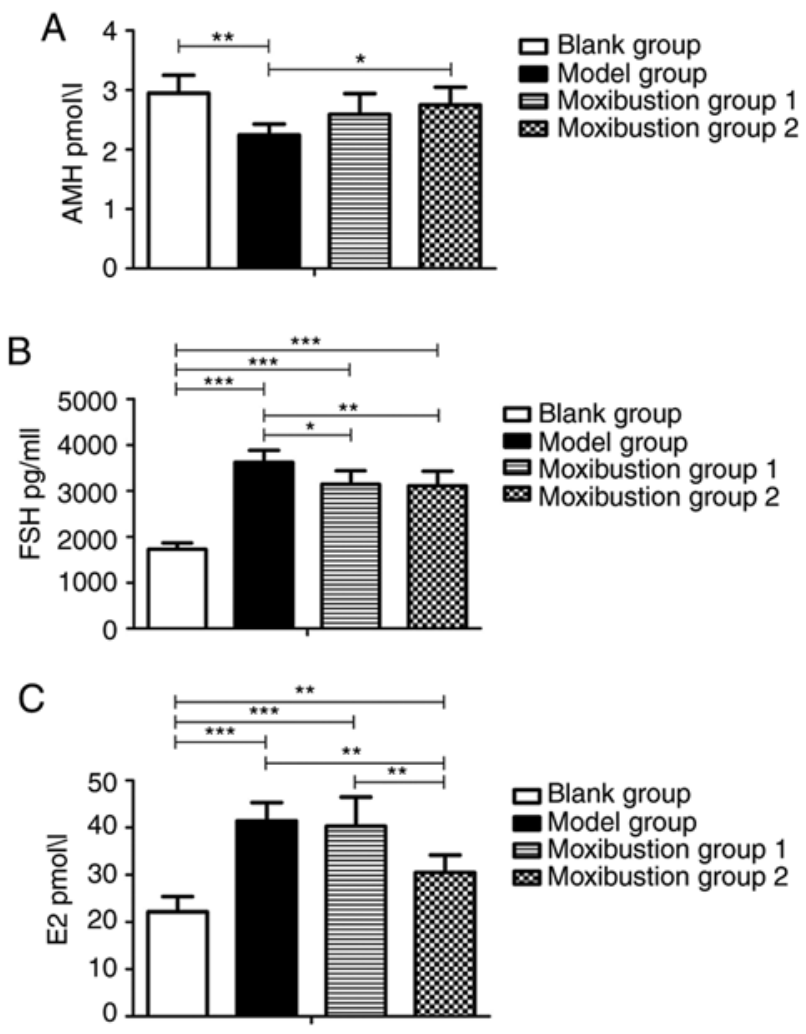

Figure 3. Effects of pre-disease or early-disease moxibustion intervention on serum levels of AMH, FSH and E2 in the different groups. Serum levels of (A) AMH, (B) FSH and (C) E2. Values are expressed as the mean \pm standard deviation. ${ }^{*} \mathrm{P}<0.05,{ }^{* *} \mathrm{P}<0.01,{ }^{* * *} \mathrm{P}<0.001$. AMH, anti-mullerian hormone; FSH, follicle-stimulating hormone; E2, estradiol.

observed between moxibustion group and model group. No difference in $\mathrm{T}$ levels was observed between the 2 moxibustion groups. Serum DHEA levels decreased in Moxibustion group 1 as compared with the model group $(24.77 \pm 3.11$ vs. $28.49 \pm 2.63 \mathrm{ng} / \mathrm{ml}, \mathrm{P}<0.05$ ), but no significant difference was observed in Moxibustion group 2 compared with the model group ( $26.10 \pm 2.36$ vs. $28.49 \pm 2.63 \mathrm{ng} / \mathrm{ml}, \mathrm{P}=0.324)$.

As presented in Fig. 4C, DHT levels significantly decreased after establishing the model $(34.20 \pm 3.60 \mathrm{vs} .18 .41 \pm 1.81 \mathrm{nmol} / 1$, $\mathrm{P}<0.001$ ), while they increased in Moxibustion group 1 and Moxibustion group 2 compared with the model group $(27.28 \pm 2.83$ vs. $18.41 \pm 1.81 \mathrm{nmol} / 1, \mathrm{P}<0.001$ and $23.19 \pm 3.77$ vs. $18.41 \pm 1.81 \mathrm{nmol} / 1, \mathrm{P}<0.05$, respectively). DHT levels were higher in Moxibustion group 1 than in Moxibustion group 2 ( $27.28 \pm 2.83$ vs. $23.19 \pm 3.77 \mathrm{nmol} / 1, \mathrm{P}=0.058$ ). No significant difference in DHT levels was observed between Moxibustion group 1 and Moxibustion group 2.

Effects of moxibustion stimulation on AR expression in the ovary with DOR. AR is an essential factor required for the androgen during follicular development. Thus, AR expression in the ovary was determined to assess whether there was any change after moxibustion treatment. As presented in Fig. 5, the relative mRNA expression of AR was decreased after establishing the model $(0.54 \pm 0.22$ vs. $0.40 \pm 0.23$, $\mathrm{P}=0.193$ ). Compared to the model group, the AR content of the ovary was increased in Moxibustion group 1 and Moxibustion group $2(0.40 \pm 0.23$ vs. $0.55 \pm 0.24, \mathrm{P}=0.130$ and
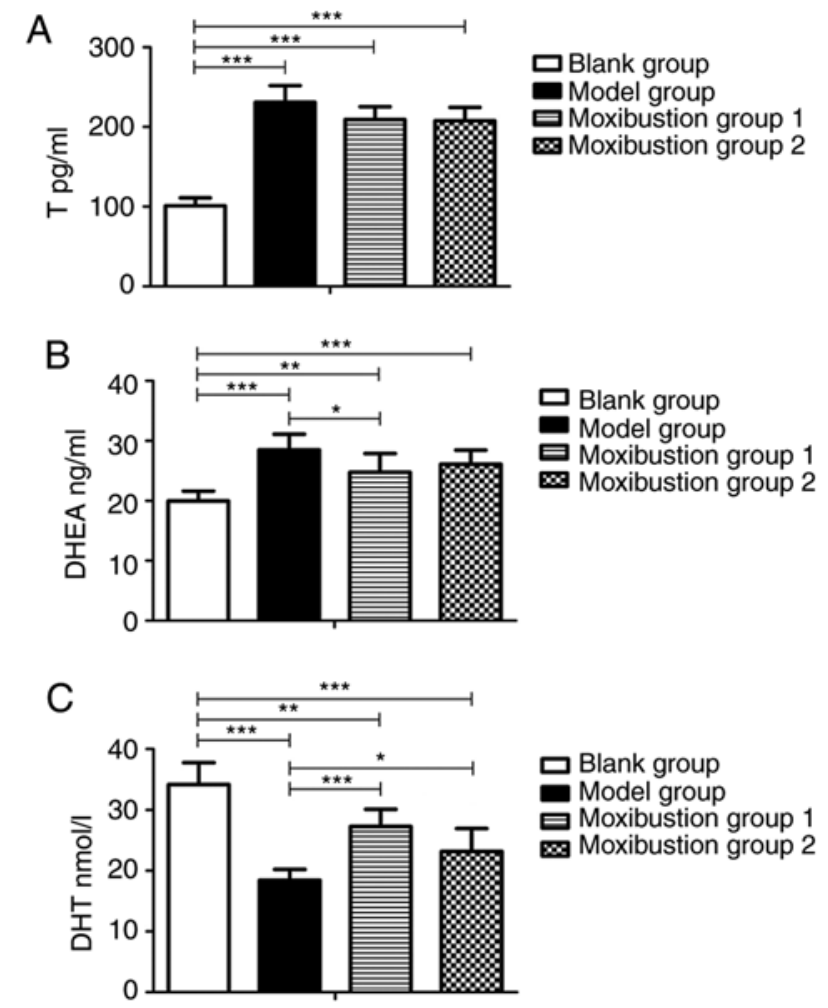

Figure 4. Effects of pre-disease or early-disease moxibustion intervention on serum levels of T, DHEA, and DHT in the different groups. Serum levels of (A) T, (B) DHEA and (C) DHT. Values are expressed as the mean \pm standard deviation. ${ }^{*} \mathrm{P}<0.05,{ }^{* *} \mathrm{P}<0.01,{ }^{* * *} \mathrm{P}<0.001$. T, testosterone; DHEA, dehydroepiandrosterone; DHT, dihydrotestosterone.

$0.40 \pm 0.23$ vs. $0.58 \pm 0.13, \mathrm{P}=0.062$, respectively). There was no significant difference in AR mRNA expression between each group.

Moxibustion stimulation induces different changes in FSHR and miR-125b expression in the ovary. Since FSHR and miRNA-125b are two different regulators of the AR-mediated signaling pathway, their expression in the ovary was analyzed to investigate whether their expression is affected by DOR and different methods of moxibustion treatment. As presented in Fig. 6A and B, the relative expression of FSHR mRNA and miR-125b decreased after establishing the model $(1.16 \pm 0.56$ vs. $0.79 \pm 0.55, \mathrm{P}=0.109$ and $2.22 \pm 1.72$ vs. $0.01 \pm 0.01, \mathrm{P}<0.001$, respectively). There was no significant difference in FSHR mRNA expression between blank group and model group. Compared to the blank and model groups, the FSHR content in the rat ovary of Moxibustion group 2 was significantly increased ( $1.16 \pm 0.56$ vs. $2.55 \pm 0.47, \mathrm{P}<0.001$ and $0.79 \pm 0.55$ vs. $2.55 \pm 0.47$, $\mathrm{P}<0.001$, respectively). However, there was no difference in FSHR mRNA in the ovaries between Moxibustion group 1 and the model group $(1.03 \pm 0.28$ vs. $0.79 \pm 0.55, \mathrm{P}=0.457)$. miR-125b was not changed in Moxibustion group 2 compared with the model group $(0.19 \pm 0.12$ vs. $0.01 \pm 0.01, \mathrm{P}=0.934)$, but a significant increase was observed in Moxibustion group 1 ( $1.18 \pm 0.53$ vs. $0.01 \pm 0.01, \mathrm{P}<0.01)$. Significant differences in FSHR and miR-125b mRNA expression were observed between Moxibustion group 1 and Moxibustion group 2 $(1.03 \pm 0.28$ vs. $2.55 \pm 0.47, \mathrm{P}<0.001$ and $1.18 \pm 0.53$ vs. $0.19 \pm 0.12$, $\mathrm{P}<0.01$, respectively). 


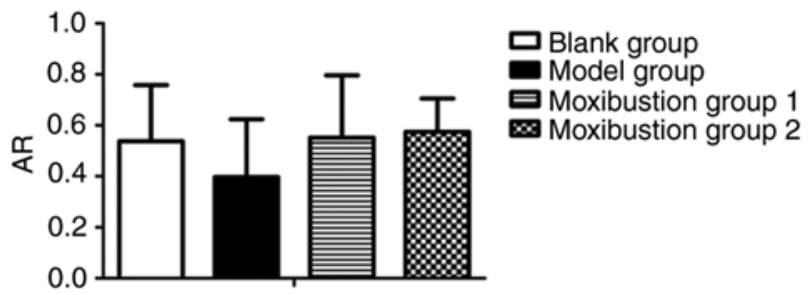

Figure 5. Effects of pre-disease or early-disease moxibustion intervention on the relative expression of AR in the ovary. Rat $18 \mathrm{~S}$ rRNA was used as reference gene. The relative mRNA expression of AR is shown in the y-axis. Values are expressed as the mean \pm standard deviation. AR, androgen receptor.

\section{Discussion}

Previous studies have indicated that moxibustion is effective in improving ovarian function, but most of them focused on treatments of disease states and there is a lack of studies on 'prevention'. Wang et al (24) found that moxibustion could reduce the rate of estrus cycle disorder, improve the level of serum sex hormones and antioxidant stress in DOR rats, and the mechanism may be related to the regulation of Nrf2/HO-1 signaling pathway. Yang et al (13) found that moxibustion could improve ovary function by suppressing apoptosis events and upregulating antioxidant defenses in the natural aging ovary.

Combined with the variation rule of reproductive aging, the average fertility of females reaches a peak at the age of 25 and then begins to decrease, declining rapidly after 35 years of age (25). The reduced fertility is manifested as a reduction in the number and quality of follicles in the ovary. In addition to the natural aging of the ovary, genetic, iatrogenic, environmental and psychological factors may also cause a premature decline in ovarian function and eventually DOR in certain females $(26,27)$. DOR is a precursor of POF. The transition from DOR to POF is a gradual dynamic evolution process. If DOR cannot be treated in a timely manner and prevented from progressing, POF will develop (28-30). Therefore, certain scholars have proposed a 'peri-premature ovarian failure stage' (31) and the gradual physiological decline in the ovary and DOR occur at this stage. According to the TCM theories of 'preventing disease in healthy states' and 'preventing progression in disease states' (32), if prevention measures are applied prior to or early in the 'peri-premature ovarian failure stage', they may effectively protect and improve ovarian function. The experiments of the present study aimed to verify this hypothesis.

The present study indicated that moxibustion prior to establishing DOR in a rat model or during the early stage of model establishment may protect ovarian function and alleviate ovarian injury caused by Tripterygium wilfordii. The present experiments demonstrated that after 2 weeks of tripterygium glycosides administration, the serum levels of $\mathrm{AMH}, \mathrm{FSH}$ and E2 in rats were significantly different from those in the blank group. The rats' estrus cycle in the model group was disordered, indicating that the model was established successfully and stable. Both pre-disease and early intervention with moxibustion rescued the increase in FSH levels and the decrease in AMH levels induced by tripterygium glycosides. The E2 level in moxibustion group
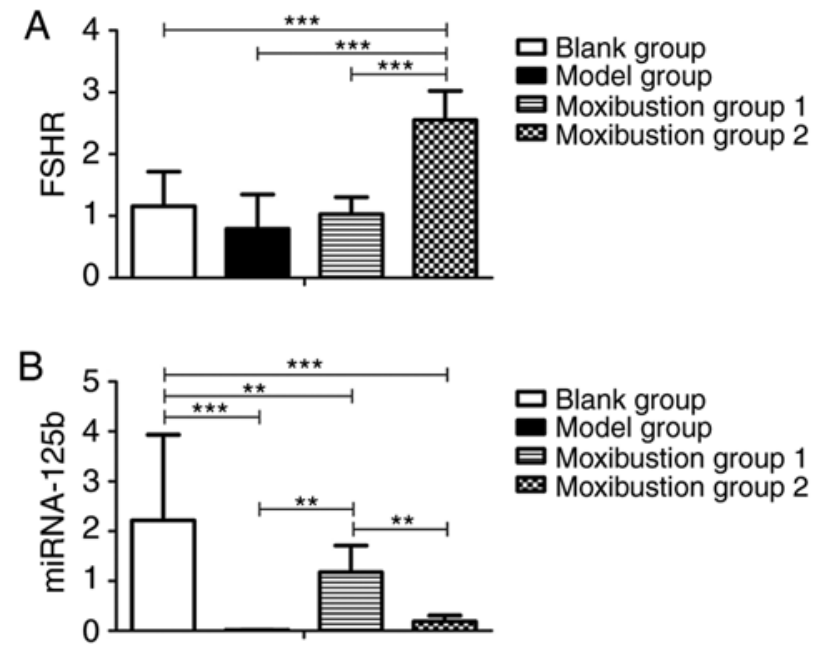

Figure 6. Pre-disease or early-disease moxibustion intervention increases FSHR and miR-125b expression in the ovary. (A) FSHR levels; rat 18S rRNA was used as reference gene. The relative mRNA expression of FSHR is shown in the $y$-axis and (B) miR-125b levels in the ovary of different groups, miRNA/U6 was used as reference gene. The relative miRNA expression of miR-125b is shown in the $y$-axis. Values are expressed as the mean \pm standard deviation. ${ }^{* *} \mathrm{P}<0.01,{ }^{* * *} \mathrm{P}<0.001$. FSHR, follicle-stimulating hormone receptor; $\mathrm{miRNA} / \mathrm{miR}$, microRNA.

1 was not different from that in the model group, while the E2 level in moxibustion group 2 was significantly lower than that in the model group. AMH, FSH and E2 are common indicators to evaluate ovarian reserve and are frequently used jointly $(33,34)$. An increase in FSH levels is related to a decrease in ovarian reserve. Elevated FSH levels promote the early recruitment and growth of follicles and thus produce higher E2 levels. However, the increase in FSH in the early stage of DOR is not stable. Due to the negative feedback regulation of E2 on the secretion of $\mathrm{FSH}$, the change in E2 is earlier than that of FSH (35). Previous studies indicated that the sensitivity and specificity of AMH in predicting ovarian response are better than those of FSH and E2 $(36,37)$. The present results suggested that moxibustion intervention prior to disease onset and during the early disease stage may improve ovarian function. Of note, the effect of moxibustion treatment at the early disease stage was better than that prior to disease onset under the same treatment conditions.

The present study focused on the effect of moxibustion on the androgen balance in a rat model of DOR. The experimental results indicated that the $\mathrm{T}$ levels were reduced in both moxibustion groups. T and E2 are positively correlated. When $\mathrm{T}$ increases in vivo, the level of estradiol converted from $\mathrm{T}$ by aromatase increases, thus causing a positive correlation between the two, and the present results are consistent with those of a previously published study (38). However, the increase in serum $\mathrm{T}$ and $\mathrm{E} 2$ levels in rats with DOR were compensatory. If ovarian function was to be further reduced and decompensated, the levels of T and E2 may decrease (38). After model establishment, the serum DHT levels in rats of the model group were lower than those in the blank group, the DHT levels in the two moxibustion groups were significantly higher than those in the model group and the serum DHT levels in moxibustion group 1 were higher than those in moxibustion group 2. A total of $20 \%$ of DHT is converted from T, which is 
an indicator of androgen levels in peripheral tissues. DHT does not convert to $\mathrm{E} 2$ without aromatase and $\mathrm{T}$ is a major indicator reflecting the secretion of ovarian androgen $(39,40)$. Therefore, DHT and T may have different trends. The present study also indicated that compared with the serum DHEA level in the blank group, the serum DHEA level in the model group was higher. The serum DHEA level in moxibustion group 1 was significantly lower but the serum DHEA level in moxibustion group 2 was the same as that in the model group. DHEA cannot directly exert its role and its conversion depends on its own production and the expression levels of metabolic enzymes in tissue. Furthermore, DHEA secretion has no negative feedback regulatory mechanism; it is an unstable androgen that acts as a 'hormone buffer' (41-44). Therefore, after model establishment, higher DHEA levels may be associated with inhibition of DHEA conversion by $T$. wilfordii. However, prior to model establishment, moxibustion treatment may significantly reduce DHEA, indicating that DHEA may be one of the targets, revealing a mechanism underlying the prevention of POF by moxibustion.

The present study also indicated that AR expression in the model group rats was lower than that prior to model establishment, while AR expression in the two moxibustion groups was higher than that in the model group. AR is an important essential in androgen-mediated signaling pathways. Studies have indicated that the female model of overall AR knockout exhibited reproductive defects, follicular development disorders and POF $(45,46)$. AR may promote follicular development through two pathways (6). First, it induces miR-125b expression, which inhibits granulose cell apoptosis, avoiding follicular atresia. Furthermore, it enhances FSHR expression, increases FSH sensitivity and promotes granulosa cell proliferation and FSH-mediated follicular growth and development. The present study also investigated the effect of moxibustion on the expression levels of FSHR and miR-125b. It was observed that the expression levels of FSHR and miR-125b in the model group were lower than those prior to model establishment, but the results for the two moxibustion groups were different. The content of miR-125b in moxibustion group 1 was significantly higher than that in the model group, while the miR-125b level in moxibustion group 2 was the same as that in the model group. However, the changes in the FSHR content were the opposite. In brief, the FSHR levels in moxibustion group 1 were the same as those in the model group, while the FSHR levels in moxibustion group 2 were significantly higher than those in the model group and even significantly higher than those in the blank group. Therefore, it may be speculated that moxibustion intervention prior to model establishment and at the early stage of model establishment may improve ovarian function via modulating different AR signaling pathways. Moxibustion treatment prior to model establishment is a preventive measure before disease onset and its function may be related to the delayed follicular atresia; moxibustion treatment at the early stage of model establishment is a treatment method for the early disease stage and its function may be related to an increase of FSH sensitivity. Therefore, it may explain why the outcomes of moxibustion group 1 and moxibustion group 2 were different even though the moxibustion regimens, sites and degree of stimulation were the same for the two moxibustion groups. Changes occurred in the acupoints' functional status after disease onset and action pathways change accordingly, which manifested as 'enhanced' acupoint functions, resulting in 'large responses to small stimulations' $(47,48)$. Studies have indicated that acupuncture also has a regulatory function on the rat HPOA under physiological conditions (49), which may explain why the FSHR levels in the moxibustion group 2 were significantly higher than those in the blank group of the present study.

A limitation of the study was that no placebo moxibustion was performed in a designated sham group. This problem will be addressed in in future studies. A higher concentration of chloral hydrate was more likely to cause abdominal inflammation, which could affect the ovaries that were harvested. Therefore, chloral hydrate with concentration of $<5 \%$ may be used for anesthesia in future studies.

In summary, the present study suggested that moxibustion intervention both prior to disease onset and at the early disease stage improved ovarian function and their protective roles are associated with AR-mediated androgen balance. However, the effects and mechanisms of the two moxibustion interventions are different. It may be speculated that to achieve the same ovarian protection effect, the treatment course of moxibustion prior to disease onset should be longer than that of moxibustion at the early disease stage; however, the appropriate duration of treatment and the time-effect relationship require to be studied further.

\section{Acknowledgements}

Not applicable.

\section{Funding}

The present study was supported by the National Natural Science Foundation of China (grant nos. 81603674, 81873371 and 81804179), the Natural Science Foundation of Jiangsu Province of China (grant no. BK20161049), and Nanjing University of Chinese Medicine 'Construction Project of Superior Nursing Discipline in Jiangsu Universities' (grant no. 2019YSHL057).

\section{Availability of data and materials}

The datasets used and/or analyzed during the current study are available from the corresponding author upon reasonable request.

\section{Authors' contributions}

$\mathrm{XJ}$ was responsible for experimental design, and was a major contributor in writing the manuscript. JC, JS and YM were responsible for collecting and analyzing data. XL, QL, HB and YL were responsible for moxibustion and index detection. YX was responsible for experimental design and paper revision. XJ and JS confirm the authenticity of all the raw data. All authors have read and approved the final manuscript.

\section{Ethics approval and consent to participate}

All experimental procedures were approved by the Animal Care and Use Committee of Nanjing University of Chinese Medicine (Nanjing, China; no. ACU170709). 


\section{Patient consent for publication}

Not applicable.

\section{Competing interests}

The authors declare that they have no competing interests.

\section{References}

1. Devine K, Mumford SL, Wu M, DeCherney AH, Hill MJ and Propst A: Diminished ovarian reserve in the United States assisted reproductive technology population: Diagnostic trends among 181,536 cycles from the Society for assisted reproductive technology clinic outcomes reporting system. Fertil Steril 104: 612-619.e3, 2015.

2. Greene AD, Patounakis G and Segars JH: Genetic associations with diminished ovarian reserve: A systematic review of the literature. J Assist Reprod Genet 31: 935-946, 2014.

3. Zhao M, Feng F, Chu C, Yue W and Li L: A novel EIF4ENIF1 mutation associated with a diminished ovarian reserve and premature ovarian insufficiency identified by whole-exome sequencing. J Ovarian Res 12: 119, 2019.

4. Gleicher N, Kim A, Weghofer A, Kushnir VA, Shohat-Tal A, Lazzaroni E, Lee HJ and Barad DH: Hypoandrogenism in association with diminished functional ovarian reserve. Hum Reprod 28: 1084-1091, 2013.

5. Vodo S, Bechi N, Petroni A, Muscoli C and Aloisi AM Testosterone-induced effects on lipids and inflammation. Mediators Inflamm 2013: 183041, 2013.

6. Sen A, Prizant H, Light A, Biswas A, Hayes E, Lee HJ, Barad D, Gleicher N and Hammes SR: Androgens regulate ovarian follicular development by increasing follicle stimulating hormone receptor and microRNA-125b expression. Proc Natl Acad Sci USA 111: 3008-3013, 2014.

7. Prizant $\mathrm{H}$, Gleicher $\mathrm{N}$ and Sen A: Androgen actions in the ovary: Balance is key. J Endocrinol 222: R141-R151, 2014.

8. Shi XL, Zhao C, Yang S, Hu XY and Liu SM: Moxibustion reduces ovarian granulosa cell apoptosis associated with perimenopause in a natural aging rat model. Evid Based Complement Alternat Med 2015: 742914, 2015.

9. Zhang CR, Deng JL, Zhu WN, Miao MX, Shen WW, Cao SJ and Tang Y: Involvement of PI 3 K/Akt/mTOR signaling in protective effects of moxibustion for premature ovarian failure in rats. Zhen Ci Yan Jiu 43: 75-79, 2018 (In Chinese).

10. Wu S and Yan J: Clinical observation on premature ovarian failure by warming acupuncture at Zusanli (ST 36) and Guanyuan (CV 4) combined with ginger moxibustion at Baliao points. Zhongguo Zhen Jiu 38: 1267-1271, 2018 (In Chinese).

11. Zhu S, Wang Y, Chang X, Chen H and Jin X: The protective effect of pre-moxibustion on reproductive hormones profile of rats with tripterygium glycosides-induced ovarian damage. Complement Med Res 27: 401-409, 2020.

12. Shen J, Shen M, Li Z, Zhang R, Li X and Ai B: Effects of moxibustion at Shenshu (BL 23) on level of sex hormones and AMH in sub-health peri-menopausal women. Zhongguo Zhen Jiu 37: 381-385, 2017 (In Chinese)

13. Yang X, Wang W, Zhang Y, Wang J and Huang F: Moxibustion improves ovary function by suppressing apoptosis events and upregulating antioxidant defenses in natural aging ovary. Life Sci 229: 166-172, 2019.

14. Zhang Y, Yu B, Chen J, Zhao Z, Wang Jiali, Huang F, Lin Y, Wang $M$, Zhang $Y$ and Wei B: Effects of acupuncture on $\mathrm{PI} 3 \mathrm{~K} / \mathrm{Akt} / \mathrm{mTOR}$ signaling pathway in rats with premature ovarian failure. Zhongguo Zhen Jiu 35: 53-58, 2015 (In Chinese).

15. Xia L and Xia Y: Clinical research and the effect mechanism on premature ovarian failure treated with acupuncture in recent 20 years. Zhongguo Zhen Jiu 38: 5653-5670, 2018 (In Chinese)

16. Zhou XL, Xu JJ, Ni YH, Chen XC, Zhang HX, Zhang XM, Liu WJ, Luo LL and Fu YC: SIRT1 activator (SRT1720) improves the follicle reserve and prolongs the ovarian lifespan of diet-induced obesity in female mice via activating SIRT1 and suppressing mTOR signaling. J Ovarian Res 7: 97, 2014.

17. Ma M, Chen XY, Gu C, Xiao XR, Guo T and Li B: Biochemical changes of oxidative stress in premature ovarian insufficiency induced by tripterygium glycosides. Int J Clin Exp Pathol 7: $8855-8861,2014$.
18. Zhang T, Yan D, Yang Y, Ma A, Li L, Wang Z, Pan Q and Sun Z: The comparison of animal models for premature ovarian failure established by several different source of inducers. Regul Toxicol Pharmacol 81: 223-232, 2016

19. Guo Y and Fang JQ: Experimental acupuncture. Beijing: China Traditional Medicine Press, pp402-407, 2012.

20. Machholz E, Mulder G, Ruiz C, Corning BF and Pritchett-Corning KR: Manual restraint and common compound administration routes in mice and rats. J Vis Exp: 2771, 2012.

21. Buerge $\mathrm{T}$ and Weiss T: The laboratory mouse: Handling and restraint. Elsevier, pp517-526, 2004.

22. Morton DB and Griffiths PH: Guidelines on the recognition of pain, distress and discomfort in experimental animals and an hypothesis for assessment. Vet Rec 116: 431-436, 1985.

23. Livak KJ and Schmittgen TD: Analysis of relative gene expression data using real-time quantitative PCR and the 2(-Delta Delta C(T)) method. Methods 25: 402-408, 2001.

24. Wang Q, Lu G, Xie Z, Xie ZJ, Li HX and Shen MH: Effect of moxibustion on Nrf2/HO-1 signaling pathway in rats with diminished ovarian reserve. Zhongguo Zhen Jiu 41: 53-58, 2021 (In Chinese).

25. Li X and Xu CJ: Diseases of the female reproductive system 1nd edition. Beijing: People's Medical Publishing House, pp1-4, 2015.

26. Galey-Fontaine J, Cédrin-Durnerin I, Chaïbi R, Massin N and Hugues JN: Age and ovarian reserve are distinct predictive factors of cycle outcome in low responders. Reprod Biomed Online 10: 94-99, 2005.

27. Shatavi SV, Llanes B and Luborsky JL: Association of unexplained infertility with gonadotropin and ovarian antibodies. Am J Reprod Immunol 56: 286-291, 2006.

28. Cohen J, Chabbert-Buffet N and Darai E: Diminished ovarian reserve, premature ovarian failure, poor ovarian responder-a plea for universal definitions. J Assist Reprod Genet 32: 1709-1712, 2015.

29. Li HF and Tan Y: Discussion on the prevention and treatment of ovarian reserve function decline from the idea of treating pre-disease. J Tradit Chin Med 31: 155-157, 2016.

30. Huang XC, Cao XC and Wang XY: Evaluation and revision of TCM guidelines on premature ovarian failure. Henan Tradit Chin Med 39: 82-86, 2019.

31. Liao HH, Zhao Y and Shi Y: ZHANG Yu-zhen's ideas and approaches concerning treatment of premature ovarian failure. Glob Tradit Chin Med 8: 780-782, 2015.

32. Meng JC and Wang XH: Internal classic. Plain questions 1nd edition. Shanghai: Shanghai Science and Technology Press, pp12-20, 2019.

33. Xu H, Zeng L, Yang R, Feng Y, Li R and Qiao J: Retrospective cohort study: AMH is the best ovarian reserve markers in predicting ovarian response but has unfavorable value in predicting clinical pregnancy in GnRH antagonist protocol. Arch Gynecol Obstet 295: 763-770, 2017.

34. Zebitay AG, Cetin O, Verit FF, Keskin S, Sakar MN, Karahuseyinoglu S, Ilhan G and Sahmay S: The role of ovarian reserve markers in prediction of clinical pregnancy. J Obstet Gynaecol 37: 492-497, 2017.

35. Du EQ, Gao X and Shang L: Assessments of anti-mullerian hormone combining with FSH and FSH/LH in the advanced maternal women during the reproductive year with ovarian reserve. Med J West China 30: 701-703, 2018.

36. Zhang H, Luo Q, Lu X, Yin N, Zhou D, Zhang L, Zhao W, Wang D, Du P, Hou Y, et al: Effects of hPMSCs on granulosa cell apoptosis and AMH expression and their role in the restoration of ovary function in premature ovarian failure mice. Stem Cell Res Ther 9: 20, 2018.

37. Lunding SA, Aksglaede L, Anderson RA, Main KM, Juul A, Hagen CP and Pedersen AT: AMH as predictor of premature ovarian insufficiency: A longitudinal study of 120 turner syndrome patients. J Clin Endocrinol Metab 100: E1030-E1038, 2015.

38. Song JM: Women testosterone levels and ovarian reserve evaluation index and the correlation of IVF outcome studies. Kunming: Kunming Medical University, pp26-27, 2016.

39. Walters KA and Handelsman DJ: Role of androgens in the ovary. Mol Cell Endocrinol 465: 36-47, 2018.

40. Gleicher N, Weghofer A, Lee IH and Barad DH: FMR1 genotype with autoimmunity-associated polycystic ovary-like phenotype and decreased pregnancy chance. PLoS One 5: e15303, 2010.

41. Qin JC, Fan L and Qin AP: The effect of dehydroepiandrosterone (DHEA) supplementation on women with diminished ovarian reserve (DOR) in IVF cycle: Evidence from a meta-analysis. J Gynecol Obstet Hum Reprod 46: 1-7, 2017. 
42. Burger HG: Androgen production in women. Fertil Steril 77 (Suppl 4): S3-S5, 2002.

43. Labrie F and Labrie C: DHEA and intracrinology at menopause, a positive choice for evolution of the human species. Climacteric 16: 205-213, 2013.

44. Gleicher N and Barad DH: Dehydroepiandrosterone (DHEA) supplementation in diminished ovarian reserve (DOR). Reprod Biol Endocrinol 9: 67, 2011.

45. Walters KA, Allan CM, Jimenez M, Lim PR, Davey RA, Zajac JD, Illingworth $\mathrm{P}$ and Handelsman DJ: Female mice haploinsufficient for an inactivated androgen receptor (AR) exhibit age-dependent defects that resemble the AR null phenotype of dysfunctional late follicle development, ovulation, and fertility. Endocrinology 148: 3674-3684, 2007.

46. Walters KA, Middleton LJ, Joseph SR, Hazra R, Jimenez M, Simanainen U, Allan CM and Handelsman DJ: Targeted loss of androgen receptor signaling in murine granulosa cells of preantral and antral follicles causes female subfertility. Biol Reprod 87: 151, 2012.
47. Zhu B: The plasticity of acupoint. Zhongguo Zhen Jiu 35: 1203-1208, 2015 (In Chinese).

48. Xu B and Han X: Discussion on primordial and sensitized states of acupuncture points. Zhen Ci Yan Jiu 43: 273-276, 2018 (In Chinese)

49. Zhu H, Nan S, Suo C, Zhang Q, Hu M, Chen R, Wan J, Li M, Chen $\mathrm{J}$ and Ding M: Electro-acupuncture affects the activity of the hypothalamic-pituitary-ovary axis in female rats. Front Physiol 10: 466, 2019.

(i) (9) This work is licensed under a Creative Commons Attribution-NonCommercial-NoDerivatives 4.0 International (CC BY-NC-ND 4.0) License. 